\title{
Diffusing-wave spectroscopy of nonergodic media ${ }^{1}$
}

\author{
F. Scheffold \\ University of Fribourg, CH-1700 Fribourg, Switzerland \\ S. E. Skipetrov \\ Department of Physics, Moscow State University, 119899 Moscow, Russia \\ S. Romer \\ University of Fribourg, CH-1700 Fribourg, Switzerland \\ Polymer Institute, ETH Zurich, CH-8092 Zurich, Switzerland \\ P. Schurtenberger \\ University of Fribourg, CH-1700 Fribourg, Switzerland
}

(October 29, 2018)

\begin{abstract}
We introduce an elegant method which allows the application of diffusing-wave spectroscopy (DWS) to nonergodic, solidlike samples. The method is based on the idea that light transmitted through a sandwich of two turbid cells can be considered ergodic even though only the second cell is ergodic. If absorption and/or leakage of light take place at the interface between the cells, we establish a so-called "multiplication rule", which relates the intensity autocorrelation function of light transmitted through the double-cell sandwich to the autocorrelation functions of individual cells by a simple multiplication. To test the proposed method, we perform a series of DWS experiments using colloidal gels as model nonergodic media. Our experimental data are consistent with the theoretical predictions, allowing quantitative characterization of nonergodic media and demonstrating the validity of the proposed technique.
\end{abstract}

\section{INTRODUCTION}

Diffusing-wave spectroscopy (DWS) [1] is an extension of "conventional" dynamic light scattering (DLS) [8,9] to the multiple scattering regime. The basic idea of DWS is to use the autocorrelation function $g_{2}(\tau)=$ $\langle I(t) I(t+\tau)\rangle_{T} /\langle I(t)\rangle_{T}^{2}$ of the light intensity $I(t)$ scattered by a turbid medium to study the dynamics of scatterers in the medium. Here $\langle\cdots\rangle_{T}$ denotes time averaging which can differ from the ensemble averaging $\langle\cdots\rangle_{E}$. Introduced in 1987 [1], the technique of DWS has rapidly evolved in recent years, and is currently applied to study various types of turbid media, such as

\footnotetext{
${ }^{1}$ Accepted for publication in Physical Review E.
}

colloidal suspensions [1 6, 10, particle gels and ceramic green bodies [11 15, emulsions [16 18, foams [19 23], granular 24,25, and biological 26 28] media. It has been demonstrated that DWS can be used to image macroscopic static and dynamic heterogeneities in turbid media 29 35. In 1995, Mason and Weitz have suggested that the motion of colloidal particles, characterized by DWS, can be directly related to the viscoelasticity of the surrounding medium (the corresponding experimental technique is sometimes called "DWS-microrheology") [36. Much attention has been paid to this approach since it could provide fast and non-invasive access to viscoelastic properties of numerous materials, thereby opening a large new field of potential applications for DWS [14, 37 39].

One of the main reasons for the remarkable success of DWS is the availability of a relatively simple and reliable theoretical model which describes the experimental data in all practically important cases. The theoretical model of DWS is based on the diffusion approximation [3,4. It applies equally well to both statistically homogeneous and heterogeneous media (media with inclusions, scatterer flows, etc.). Absorption of light, reflection of scattered waves at the sample boundaries, modulation of the source intensity, as well as various types of scatterer motion (Brownian [3, 4] and sub-brownian [40] motion, laminar 41 43 and turbulent 44 flows, etc.) can be taken into account within the framework of the diffusion model. Recently, the theory of DWS has been extended to nonlinear random media [45,46].

An important condition for the applicability of the existing diffusion theory to DWS experiments is the ergodicity of the turbid medium under investigation. Indeed, ensemble-averaged quantities are commonly calculated theoretically, while it is the time averaging which is most easily obtained in experiments. Thus, $\langle\cdots\rangle_{E}=\langle\cdots\rangle_{T}$ is required for the experimental data to be described by the theory. If the light-scattering sample is nonergodic (say, the sample or some part of it is solid-like) additional efforts, e.g. translational or rotational motion of 
the sample during the measurement, are necessary in order to obtain $\langle\cdots\rangle_{E}$ 29,33, 35. Similar arguments hold for the role of nonergodicity in standard DLS experiments 4753 .

In the present paper we propose a novel, elegant and simple way of performing diffusing-wave spectroscopy of nonergodic media 11,55. The method is based on the idea that light transmitted trough a sandwich of two turbid cells can be considered ergodic even if only the second cell is ergodic. We show that the resulting transmitted multiple-scattered intensity is ergodic despite the nonergodicity of random medium in the first cell. Consequently, the usual DWS theory applies to the description of the intensity autocorrelation function $g_{2}(\tau)$. Moreover, the double-cell sample can be constructed in a way that the presence of the second, ergodic cell does not obscure the light-scattering signal of the nonergodic medium under investigation. In order to simplify the interpretation of the experimental data, we introduce a so-called "multiplication rule". Namely, we show that if a significant attenuation of diffuse waves occurs at the interface between the cells due to absorption and/or leakage of light in transverse directions, the ensemble-averaged field [56] autocorrelation function $g_{1}^{(2)}\left(L_{1}, L_{2}, \tau\right)$ of the doublecell setup equals a product of autocorrelation functions $g_{1}^{(1)}\left(L_{1}, \tau\right)$ and $g_{1}^{(1)}\left(L_{2}, \tau\right)$ corresponding to the individual cells [from here on, we use superscripts (1) and (2) to denote the autocorrelation functions corresponding to single- and double-cell samples, respectively; $L_{1}$ and $L_{2}$ are the thicknesses of the cells]. This allows a full DWS study of the turbid media in the first cell despite its nonergodicity. Due to its simplicity and high statistical accuracy, our method is ideally suited for the study of turbid gels, colloidal glasses, and other nonergodic multiplescattering media [11, 12, 15, 55]. The method also extends the applicability of the above-mentioned microrheology approach 36, 37 to an important class of solid-like complex materials where the motion of colloidal tracer particles is highly constrained.

The paper is organized as follows. In the next section, we provide a brief review of known experiments in the field of DLS by nonergodic media. We describe the methods used to overcome the problem of nonergodicity, and discuss the applicability of similar methods in DWS experiments. In the second part of the section, we introduce an original, novel method to deal with nonergodicity in DWS and reveal the conditions under which the method can be applied. Section III is devoted to the theoretical model of DWS in a double-layer medium. Starting from the diffusion equation for the field autocorrelation function, we obtain an expression for the autocorrelation function of light transmitted through a sandwich of two turbid cells, separated by a nonscattering but perhaps absorbing wall. Section IV introduces the so-called multiplication rule, and reveals the conditions of its applicability. In Sec. \ we describe our experimental setup, and Sec. VI is devoted to the discussion of the main results of our experiments. First, we have performed model experiments to test the validity of our theoretical model, and to ensure that the experimental setup is adequately described by the theory. In these experiments, no attenuation occurs on passage of light through the wall separating the light-scattering cells, and the multiplication rule does not apply. Next, we show how our method can be applied to an important case of considerable leakage of light at the inter-cell wall. This situation is the most interesting for practical applications, since the multiplication rule implies that the autocorrelation function $g_{1}^{(1)}\left(L_{1}, \tau\right)$ corresponding to the first layer can be obtained simply by dividing $g_{1}^{(2)}\left(L_{1}, L_{2}, \tau\right)$ measured for the two-cell setup by $g_{1}^{(1)}\left(L_{2}, \tau\right)$ measured for the second cell taken alone. In addition, our second sample is highly asymmetric: the first layer is of significant optical thickness and has a relatively short correlation decay time $\tau_{1}$, while the optical thickness of the second one is moderate and its correlation decay time $\tau_{2} \gg \tau_{1}$. This prevents the light-scattering signal of the first, nonergodic cell from being obscured by the second cell. In Sec. VII we discuss different aspects of application and optimization of the two-cell technique in practice. We summarize our main results in Sec. VIII.

\section{DYNAMIC LIGHT SCATTERING BY NONERGODIC MEDIA}

In nonergodic random media, the scatterers are localized near fixed average positions, probing only a small fraction of their possible spatial configurations by thermal motion. As a consequence, the measured timeaveraged quantities (such as the scattered intensity or its autocorrelation function) differ from the ensembleaveraged ones. Experimentally, one finds that a series of (time-averaged) measurements on a given sample yields a set of different results, each being of limited use for the characterization of the medium (see Fig. 1).

\section{A. Concepts to deal with nonergodicity in dynamic light scattering}

For diluted, nonergodic samples several methods have been applied to properly average the data obtained in DLS experiments [47 53. A good comparative review of some of these methods can be found in Refs. [50] and [51]. The most direct method of performing ensemble averaging of scattered light for nonergodic samples is based on the idea of collecting light scattered by different parts of the sample, thus performing the "real" ensemble averaging [50]. Experimentally, the sample is slowly moved or rotated, while the autocorrelation function of the scattered intensity $g_{2}(\tau)$ is collected. 


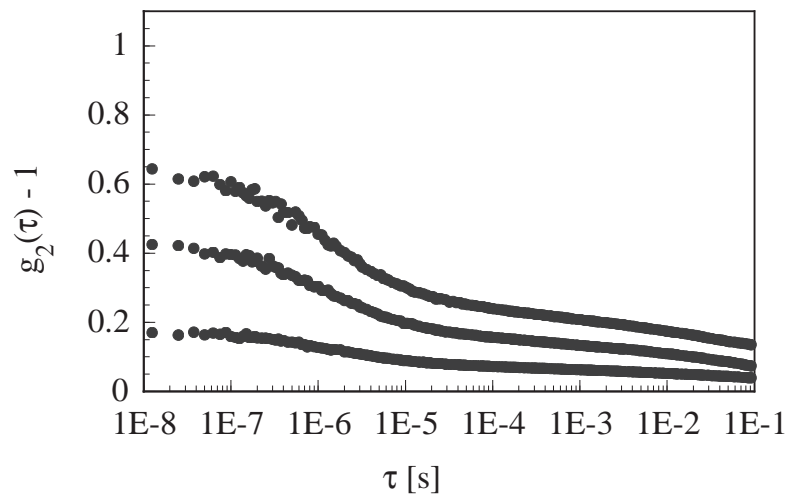

FIG. 1. Typical light scattering signal of a non-ergodic system. Repeated (time averaged) measurements of $g_{2}(\tau)-1$ lead to non-reproducible results, each being of limited use for the characterization of the system. Over the measurement time (typically a few minutes), the time and ensemble averages of the fluctuations of the scattered light intensity are not the same.

Obviously, this leads to an additional decay of $g_{2}(\tau)$, which becomes increasingly rapid with increase of the translation velocity or the rotation frequency [50]. The rotation/translation method can be extended to concentrated, turbid suspensions without any particular difficulties, and it has been actually employed in recent experiments on diffusing-wave imaging [34,35]. It has, however, an important disadvantage of experimental complexity (translation or rotation of the sample is required). Besides, it is not well suited for fragile turbid systems (such as gels), since moving or rotating the sample can lead to its disturbance or even breakdown.

Pusey and Van Megen [47] have proposed a method of obtaining the ensemble-averaged intensity autocorrelation function from a combination of static and dynamic experiments. The idea is to measure the autocorrelation function of scattered intensity for a single sample orientation, and to subsequently rotate/translate the sample rapidly in order to obtain the average intensity for a given scattering angle $[47,49,57]$. Recently, an extension of the above method to DWS experiments has been developed [58.

In general, all these approaches rely on a one dimensional motion of the sample, i.e. scanning the speckle pattern, which is not very efficient and therefore time consuming. This makes it intrinsically difficult to apply these methods to systems which evolve in time, e.g. gelling systems.

A different method of dealing with nonergodicity in DLS has been proposed in Refs. [52] and [53] (see also Ref. [54). The authors use a CCD camera to record the temporal evolution of many speckle spots simultaneously, which allows them to perform correctly both time and ensemble averages (the latter is the average over a large number of speckle spots). The method appears to be very efficient in the small-angle single-scattering regime, while its application to multiple-scattering systems is complicated by a (generally) insufficient time resolution of available CCD cameras (in DWS, fast and low-intensity speckle fluctuations are usually monitored). However, the method of Refs. [52] and [53 can be applied to study extremely slow dynamics in turbid systems where the above mentioned constraints do not apply [59].

\section{B. Two-cell technique}

To overcome the problem of nonergodicity, we prepare a sandwich consisting of two independent glass cells. The first cell contains a solid-like nonergodic medium under study, while the second cell is filled with an ergodic medium [see Fig. 2(a)]. By adjusting the concentration of scatterers in the second cell, the viscosity of the liquid where the scatterers are suspended, and the thickness of the cell, it is possible to shift the "forced" decay of the autocorrelation function due to the second cell to long correlation times $\tau$. Then $g_{1}^{(2)}\left(L_{1}, L_{2}, \tau\right)$ will exhibit a short-time decay due to the motion of scatterers in the first cell, at intermediate $\tau$ it will saturate at a plateau because of the nonergodic nature of the medium in the first cell, and finally, at long correlation times, $g_{1}^{(2)}\left(L_{1}, L_{2}, \tau\right)$ will decrease to zero due to the motion of scatterers in the second cell. Physically, the slow motion of scatterers in the second cell gently shakes and randomizes the speckle pattern of the nonergodic medium. In this way, ensemble averaging of light scattered by the first cell is accomplished, similarly to the case when the sample is moved. The time scale of this averaging can be well controlled by the scatterer motion inside the second cell, and by the thickness of the cell. The only constraint being a high enough optical thickness of the second cell, ensuring that the nonergodic intensity fluctuations produced by the first cell are averaged out upon the transmission through the second one, and that no light can pass through the latter without being scattered. The described setup offers an advantage of high statistical accuracy due to a two dimensional averaging scheme. Furthermore, no mechanical disturbance is applied to the sample, which is of particular importance when working with fragile systems.

Let us briefly discuss different aspects of time and ensemble averages in the double-cell geometry. For simplicity, we choose the intensity of light (and not its autocorrelation function) as an object of averaging, but the same arguments apply to the autocorrelation function as well. First, we consider the transmission of a plane wave through a layer of totally rigid random medium (thickness $L_{1}$, photon transport mean free path $l_{1}^{*}$ ). We find that the ensemble-averaged intensity of multiplescattered light $\langle I(\mathbf{R}, z)\rangle_{E}$ at depth $z$ and transverse position $\mathbf{R}=\{x, y\}$ is independent of $\mathbf{R}$. Meanwhile, $\langle I(\mathbf{R}, z)\rangle_{T} \equiv I(\mathbf{R}, z)$ is a random function of $\mathbf{R}$, a "fingerprint" of a given scatterer distribution in the sample. 

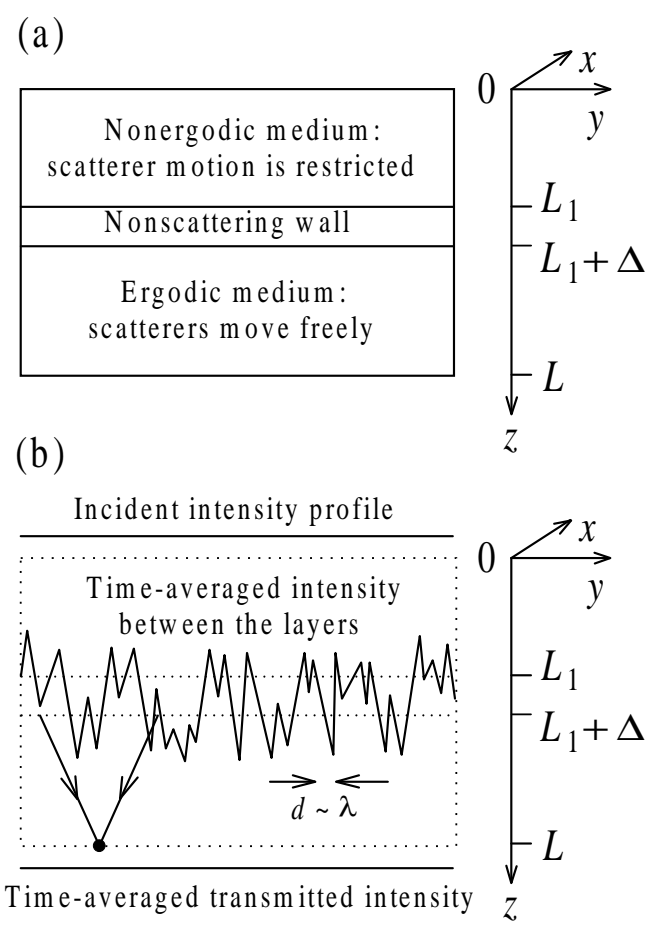

FIG. 2. Schematic illustration of the idea of the two-cell technique. (a) A cell (thickness $L_{2}$ ) containing an ergodic turbid medium is added just after the cell containing the nonergodic medium under investigation (thickness $L_{1}$ ). The cells are separated by a glass wall (thickness $\Delta$ ). The overall thickness of the sample is $L=L_{1}+\Delta+L_{2}$. (b) Time-averaged intensity profiles are shown schematically for the incident light, the multiple-scattered light at the inter-cell interface $z=L_{1}$, and for the transmitted light. Between the cells, the time-averaged intensity exhibits speckles due to the nonergodic nature of the medium in the first cell. The speckles are washed out upon the transmission through a sufficiently thick second cell.

Consequently, $\langle I(\mathbf{R}, z)\rangle_{E} \neq\langle I(\mathbf{R}, z)\rangle_{T}$, and the considered light-scattering system is nonergodic.

Let us now add a second turbid layer (thickness $L_{2}$, photon transport mean free path $l_{2}^{*}$ ) just after the first one [we neglect for a moment the thickness $\Delta$ of a glass wall separating the cells, see Fig. 2(a)]. We assume that the scatterers in the second layer are moving, and that all possible spatial configurations of scatterers are explored in course of this motion. The light intensity $I(\mathbf{R}, z, t)$ is then time-dependent, and the time-averaged intensity of light transmitted through the double-layer medium, $\langle I(\mathbf{R}, L, t)\rangle_{T}$, can be considered as a result of transmission of the speckle pattern $\left\langle I\left(\mathbf{R}, L_{1}, t\right)\right\rangle_{T}$, being specific for the given configuration of scatterers inside the first layer, through the second layer. Now we assume that the optical thicknesses of both layers are large enough $\left(L_{n} \gg l_{n}^{*}\right)$, so that the diffusion picture of wave trans- port is valid in both layers. This allows us to estimate the typical speckle size at the $z=L_{1}$ plane as $d \sim \lambda$ (see, e.g., Ref. [69]), where the wavelength of light $\lambda$ is assumed to be roughly the same in both layers and much smaller than the scattering lengths in the layers. At the same time, as long as $L_{2} \gg l_{2}^{*}$, the average intensity of light transmitted through the second layer is not sensitive to those details of the "source distribution" $I\left(\mathbf{R}, L_{1}, t\right)$ which are finer than $L_{2}$. Consequently, the speckle pattern $I\left(\mathbf{R}, L_{1}, t\right)$ will be completely washed out upon the transmission through the second layer, and $\langle I(\mathbf{R}, L, t)\rangle_{T}$ will be insensitive to the exact spatial configuration of scatterers in the first (rigid) layer, being equal to $\langle I(\mathbf{R}, L, t)\rangle_{E}$. The scattering system is then ergodic: the time and ensemble averages are equivalent. Similar arguments apply in the case when the first layer is not completely rigid, but exhibits some nonergodic dynamics. The general reason for this is the following. A given speckle spot of typical size $d \ll l_{2}^{*}$ in the $z=L_{1}$ plane can be considered as a point source of light, which produces a photon cloud spreading linearly upon the diffusion through the second layer of turbid medium. Hence, the speckle spot at $z=L_{1}$ gives contributions to the average intensity within a region of typical transverse size $L_{2}$ at the $z=L$ plane. For this reason, the intensity of light at a given point $\mathbf{R}$ of the $z=L$ plane is formed as a sum of contributions of $N \sim\left(L_{2} / d\right)^{2} \gg 1$ speckle spots located at $z=L_{1}$ [see Fig. 2(b)]. In this way, the averaging over a large number of independent speckle spots is performed. In some sense, such an averaging is equivalent to that obtained by translation (or rotation) of a single nonergodic layer. It is worthnoting, however, that in order to average over the same number $N$ of speckle spots, the sample has to be translated by a distance of order $L_{2}^{2} / d \gg L_{2}$.

\section{THEORY OF DWS IN A DOUBLE-LAYER MEDIUM}

Adding a second cell modifies the intensity autocorrelation function of transmitted light, which now exhibits an additional decay. In order to interpret properly the experimental data, we need a theoretical model describing the autocorrelation function in a double-layer turbid medium. In this section, we show that such a model can be readily constructed, provided that the transport of light is diffusive in both layers.

Consider a slab situated between the planes $z=0$, $z=L=L_{1}+\Delta+L_{2}$, and consisting of two layers of turbid media (thicknesses $L_{1}$ and $L_{2}$ ) separated by a nonscattering but perhaps absorbing wall (thickness $\Delta$, absorption coefficient $M_{a}$ ), as depicted in Fig. 2(a). $L_{1}$ and $L_{2}$, are assumed to be much larger than the photon transport mean free paths inside the layers, $l_{1}^{*}$ and $l_{2}^{*}$, respectively. If the slab is illuminated by a plane monochromatic wave, the field correlation function $G_{1}(\mathbf{r}, \tau)=\left\langle E(\mathbf{r}, t) E^{*}(\mathbf{r}, t+\tau)\right\rangle_{E}$ inside each turbid 
layer can be found as a solution of the diffusion equation [29,31:

$$
\left[\nabla^{2}-\alpha^{2}(\tau)\right] G_{1}(\mathbf{r}, \tau)=-\frac{3 S(\mathbf{r})}{l^{*}},
$$

where $\alpha^{2}(\tau)$ describes "attenuation" of correlation due to scatterer motion, and $S(\mathbf{r})=s_{0} \delta\left(z-z_{0}\right)$ is the source term $\left(z_{0} \approx l_{1}^{*}\right)$. The explicit form of the "attenuation" coefficient $\alpha^{2}(\tau)$ in Eq. (11) depends on the type of scatterer motion (e.g., Brownian motion, flow, etc.). One can show that $\alpha^{2}(\tau)$ is determined by the absorption length $l_{a}$ and the mean square scatterer displacement $\left\langle\Delta r(\tau)^{2}\right\rangle_{E}$ [5. 31,65$]$ :

$$
\alpha^{2}(\tau)=\frac{3}{l_{a} l^{*}}+\frac{k^{2}}{l^{* 2}}\left\langle\Delta r(\tau)^{2}\right\rangle_{E}
$$

where $k=2 \pi / \lambda$ is the wave number of light in the medium. For Brownian motion [3,, 4$],\left\langle\Delta r(\tau)^{2}\right\rangle_{E}=$ $\left\langle\Delta r(\tau)^{2}\right\rangle_{T}=6 D_{B} \tau$, and $\alpha^{2}(\tau)=3 /\left(l_{a} l^{*}\right)+6 \tau /\left(\tau_{0} l^{* 2}\right)$ with $\tau_{0}=\left(k^{2} D_{B}\right)^{-1}$, while for a directed flow one finds 411 43] $\left\langle\Delta r(\tau)^{2}\right\rangle_{E, T} \propto \tau^{2}$, and $\alpha^{2}(\tau) \propto \tau^{2}$. In the case of a gel, which is an example of nonergodic medium considered in this paper, we assume that the particles undergo a sort of arrested subdiffusive motion [11, 58, 66]:

$$
\left\langle\Delta r(\tau)^{2}\right\rangle_{E, T}=\delta^{2}\left\{1-\exp \left[-\left(\frac{\tau}{\tau_{c}}\right)^{p}\right]\right\}
$$

where $\delta$ denotes the maximum r.m.s. particle displacement, $\tau_{c}$ is a characteristic time required for this displacement to occur, and $p$ is a free parameter. We expect 11] $p \simeq 0.7 \pm 0.1$. Eqs. (2) and (3) give

$$
\alpha^{2}(\tau)=\frac{3}{l_{a} l^{*}}+\frac{(k \delta)^{2}}{l^{* 2}}\left\{1-\exp \left[-\left(\frac{\tau}{\tau_{c}}\right)^{p}\right]\right\} .
$$

The time autocorrelation function for a sample consisting of two turbid layers separated by a nonscattering wall can be found by solving Eq. (1) inside each layer, and then applying the boundary conditions at the surfaces of the medium ( $z=0$ and $z=L)$ and at the interfaces between the turbid layers and the nonscattering wall. Boundary conditions at $z=0$ and $z=L$ are of a well-known form 60 63]:

$$
\begin{aligned}
& {\left.\left[G_{1}(\mathbf{r}, \tau)-z_{1}\left(\mathbf{e}_{z} \cdot \nabla G_{1}(\mathbf{r}, \tau)\right)\right]\right|_{z=0}=0} \\
& {\left.\left[G_{1}(\mathbf{r}, \tau)+z_{2}\left(\mathbf{e}_{z} \cdot \nabla G_{1}(\mathbf{r}, \tau)\right)\right]\right|_{z=L}=0}
\end{aligned}
$$

where $\mathbf{e}_{z}$ is a unit vector parallel to the $z$-axis, $z_{n}=$ $(2 / 3) l_{n}^{*}$, and the refractive index mismatch between the scattering and transparent media is neglected. In general, a rigorous theory would require using the extrapolation lengths $z_{n}$ calculated with account for the refractive index mismatch at the sample surfaces 60 63], or deduced from the angular distribution of diffusely transmitted light 64. For our purposes, however, it is sufficient to know that $z_{n} \sim \ell_{n}^{*}$, since the actual values of $z_{n}$ are of no importance in the limit of $\alpha_{n} l_{n}^{*} \ll 1$ and $l_{n}^{*} / L_{n} \ll 1$ that we apply in the following.

Boundary conditions at the interfaces $z=L_{1}$ and $z=L_{1}+\Delta$ between turbid and nonscattering media are found by applying the condition of flux conservation, as discussed by Ripoll et. al. [70]. Neglecting the refractive index mismatch between the turbid and nonscattering media, we find [71]:

$$
\begin{aligned}
& {\left.\left[G_{1}(\mathbf{r}, \tau)+z_{1}\left(\mathbf{e}_{z} \cdot \nabla G_{1}(\mathbf{r}, \tau)\right)\right]\right|_{z=L_{1}}} \\
& \quad=\left.f(\mathbf{r})\left[G_{1}(\mathbf{r}, \tau)+z_{2}\left(\mathbf{e}_{z} \cdot \nabla G_{1}(\mathbf{r}, \tau)\right)\right]\right|_{z=L_{1}+\Delta} \\
& {\left.\left[G_{1}(\mathbf{r}, \tau)-z_{2}\left(\mathbf{e}_{z} \cdot \nabla G_{1}(\mathbf{r}, \tau)\right)\right]\right|_{z=L_{1}+\Delta}} \\
& \quad=\left.f(\mathbf{r})\left[G_{1}(\mathbf{r}, \tau)-z_{1}\left(\mathbf{e}_{z} \cdot \nabla G_{1}(\mathbf{r}, \tau)\right)\right]\right|_{z=L_{1}}
\end{aligned}
$$

with $f(\mathbf{r})$ describing the losses of energy at the interlayer wall due to absorption and/or leakage of light in transverse directions $[0 \leq f(\mathbf{r}) \leq 1$ and $f(\mathbf{r}) \equiv 1$ in the absence of both absorption and leakage]. For an infinitely wide slab (no leakage) we have [7]

$$
f=\exp \left(-M_{a} \Delta\right)\left(1-M_{a} \Delta\right)+\left(M_{a} \Delta\right)^{2} \Gamma\left(0, M_{a} \Delta\right),
$$

where $\Gamma(\ldots)$ is the incomplete gamma function. For realistic samples of finite width, leakage of light in transverse directions may be considerable. In the absence of absorption $\left(M_{a}=0\right)$, we find for a cylindrical sample of radius $R \gg l_{n}^{*}$ after averaging over the sample crossection:

$$
f=1+\frac{1}{2}\left(\frac{\Delta}{R}\right)^{2}-\frac{1}{2} \frac{\Delta}{R}\left[4+\left(\frac{\Delta}{R}\right)^{2}\right]^{1 / 2} .
$$

Averaging over the sample crossection makes $f$ independent of $\mathbf{r}$, which largely simplifies the further analysis without affecting the final result qualitatively.

In what follows, we assume that $f(\mathbf{r})$ in Eqs. (7) and (8) is given either by Eq. (9) or by Eq. (10), being independent of $\mathbf{r}$ in both cases. The case when both absorption and leakage are present can also be analyzed without any particular difficulties, but this leads to cumbersome formulas without introducing any new qualitative features. Both Eqs. (9) and (10) exhibit a monotonic decrease from $1\left(M_{a} \Delta=0\right.$ or $\left.\Delta / R=0\right)$ to $0\left(M_{a} \Delta \gg 1\right.$ or $\Delta / R \gg 1$ ). If $f=1$ (no absorption, no leakage), Eqs. (7) and (8) reduce to the well-known boundary conditions between two turbid media in a direct contact 29, 31, 32. If $f=0$ (strong absorption and/or leakage), Eqs. (7) and (8) decouple and the problem is reduced to two single-layer problems.

Equations (1) and (5)-(8) enables us to calculate the autocorrelation function of light transmitted through the double-layer system, $G_{1}(L, \tau) / G_{1}(L, 0)$, which in the absence of absorption $\left(l_{a} \rightarrow \infty\right)$ inside the turbid layers reads

$$
\begin{aligned}
& g_{1}^{(2)}\left(L_{1}, L_{2}, \tau\right)=\frac{\alpha_{1} L_{1}}{\sinh \left(\alpha_{1} L_{1}\right)} \frac{\alpha_{2} L_{2}}{\sinh \left(\alpha_{2} L_{2}\right)} \\
& \quad \times\left(1-f^{2}+2 z_{1} / L_{1}+2 z_{2} / L_{2}\right) \\
& \quad \times\left[1-f^{2}+\frac{2 \alpha_{1} z_{1}}{\tanh \left(\alpha_{1} L_{1}\right)}+\frac{2 \alpha_{2} z_{2}}{\tanh \left(\alpha_{2} L_{2}\right)}\right]^{-1},
\end{aligned}
$$


where we assumed $\alpha_{n} l_{n}^{*} \ll 1$ and $l_{n}^{*} / L_{n} \ll 1$.

In our experiments, the time-averaged autocorrelation function of the scattered intensity $g_{2}(\mathbf{r}, \tau)=$ $\langle I(\mathbf{r}, t) I(\mathbf{r}, t+\tau)\rangle_{T} /\langle I(\mathbf{r}, t)\rangle_{T}^{2}$ is measured. As the double-layer system is shown to be ergodic (see Sec. II), $g_{2}$ is related to $g_{1}$ by the Siegert relation [8]:

$$
g_{2}(\mathbf{r}, \tau)=1+\beta\left|g_{1}(\mathbf{r}, \tau)\right|^{2}
$$

where $\beta \simeq 1$ is a constant determined by the experimental setup [8,9]. Let us assume that the first layer is filled with a gel, while the second one contains a suspension of Brownian particles with a correlation decay time $\tau_{2}=\tau_{0}\left(l_{2}^{*} / L_{2}\right)^{2}$. We also assume that the characteristic correlation decay time of the first layer $\tau_{1}$ is much smaller than $\tau_{2}$. Then, it can be shown from Eqs. (11) and (12) that when the r.m.s. particle displacement $\left\langle\Delta r(\tau)^{2}\right\rangle^{1 / 2}$ approaches $\delta$ for $\tau \gg \tau_{1}$ [Eq. (3)], the autocorrelation function $g_{1}^{(2)}\left(L_{1}, L_{2}, \tau\right)$ reaches a plateau for $\tau_{1} \ll \tau \ll \tau_{2}$. The plateau height can be found from Eq. (11). As $\tau$ approaches $\tau_{2}, g_{1}^{(2)}\left(L_{1}, L_{2}, \tau\right)$ continues to decrease.

\section{MULTIPLICATION RULE}

The autocorrelation function of light transmitted through a single layer of turbid medium can be found [31] from Eq. (11) with boundary conditions (5) and (6), and since for $\alpha l^{*} \ll 1$ and $L \gg l^{*}$ the solution reads

$$
g_{1}^{(1)}(L, \tau)=\frac{\alpha L}{\sinh (\alpha L)}
$$

We can rewrite Eq. (11) as

$$
g_{1}^{(2)}\left(L_{1}, L_{2}, \tau\right)=g_{1}^{(1)}\left(L_{1}, \tau\right) \times g_{1}^{(1)}\left(L_{2}, \tau\right) \times F,
$$

where

$$
\begin{aligned}
F & =\left(1-f^{2}+2 z_{1} / L_{1}+2 z_{2} / L_{2}\right) \\
& \times\left[1-f^{2}+\frac{2 \alpha_{1} z_{1}}{\tanh \left(\alpha_{1} L_{1}\right)}+\frac{2 \alpha_{2} z_{2}}{\tanh \left(\alpha_{2} L_{2}\right)}\right]^{-1} .
\end{aligned}
$$

Hence, the autocorrelation function of light transmitted through the double-layer sample is given by a product of autocorrelation functions of individual layers, times some function $F$ which describes the coupling between the layers. By adjusting the parameters of the experimental setup, $F \simeq 1$ can be achieved, and then the following multiplication rule will hold:

$$
g_{1}^{(2)}\left(L_{1}, L_{2}, \tau\right) \simeq g_{1}^{(1)}\left(L_{1}, \tau\right) \times g_{1}^{(1)}\left(L_{2}, \tau\right) .
$$

To reveal the conditions of validity of the multiplication rule $(16)$, we consider separately the cases of low $(f \simeq 1)$ and considerable $(f<1)$ losses of light at the inter-layer interface.
If $f \simeq 1$, Eq. (15) becomes

$$
\begin{aligned}
F & \simeq\left[\frac{L_{1}}{l_{1}^{*}}+\frac{L_{2}}{l_{2}^{*}}\right] \\
& \times\left[\frac{L_{1}}{l_{1}^{*}} \frac{\alpha_{2} L_{2}}{\tanh \left(\alpha_{2} L_{2}\right)}+\frac{L_{2}}{l_{2}^{*}} \frac{\alpha_{1} L_{1}}{\tanh \left(\alpha_{1} L_{1}\right)}\right]^{-1},
\end{aligned}
$$

which reduces to unity only if $\alpha_{1} L_{1} \ll 1$ and $\alpha_{2} L_{2} \ll 1$, or $L_{1} / l_{1}^{*} \gg L_{2} / l_{2}^{*}$ and $\alpha_{2} L_{2} \ll 1$, or $L_{1} / l_{1}^{*} \ll L_{2} / l_{2}^{*}$ and $\alpha_{1} L_{1} \ll 1$. In other words, for the multiplication rule (16) to hold the optical thickness of e.g. the first layer should be much greater than that of the second one, while $g_{1}^{(1)}\left(L_{2}, \tau\right) \simeq 1$ for the latter.

In the presence of losses at the inter-layer interface, $f<1$ and Eq. (15) yields $F \simeq 1$ if $L_{n} / l_{n}^{*} \gg 1 /\left(1-f^{2}\right)$ and $\alpha_{n} l_{n}^{*} / \tanh \left(\alpha_{n} L_{n}\right) \ll 1-f^{2}$. To give an example, suppose that $50 \%$ of wave energy is lost on crossing the interlayer interface once $(f=0.5)$. This gives $L_{n} / l_{n}^{*} \gg 4 / 3$ and $\alpha_{n} l_{n}^{*} / \tanh \left(\alpha_{n} L_{n}\right) \ll 3 / 4$. The latter conditions are commonly satisfied in typical DWS experiments, making the application of the multiplication rule rather practical.

It is worthwhile to note that there exists a different, less rigorous, but more transparent way of establishing the multiplication rule. Adopting the path-integral picture of light propagation through the double-layer medium [2 -4, we can write the field autocorrelation function of transmitted light as

$$
\begin{aligned}
& g_{1}^{(2)}\left(L_{1}, L_{2}, \tau\right)=\int_{0}^{\infty} d s_{1} \int_{0}^{\infty} d s_{2} P_{2}\left(s_{1}, s_{2}\right) \\
& \quad \times \exp \left[-\frac{1}{2}\left\langle\Delta \varphi^{2}(\tau)\right\rangle_{s_{1}}-\frac{1}{2}\left\langle\Delta \varphi^{2}(\tau)\right\rangle_{s_{2}}\right]
\end{aligned}
$$

where $P_{2}\left(s_{1}, s_{2}\right)$ is the relative weight of paths consisting of segments of overall lengths $s_{1}$ and $s_{2}$ inside the first and the second layers, respectively. The variances of phase differences $\left\langle\Delta \varphi^{2}(\tau)\right\rangle_{s_{n}}$ describe the dephasing of light inside the layers. For a single layer of thickness $L$ one has 2 ,

$$
g_{1}^{(1)}(L, \tau)=\int_{0}^{\infty} d s P_{1}(s) \exp \left[-\frac{1}{2}\left\langle\Delta \varphi^{2}(\tau)\right\rangle_{s}\right] .
$$

Obviously, if the variables $s_{1}$ and $s_{2}$ can be considered independent, $P_{2}\left(s_{1}, s_{2}\right)=P_{1}\left(s_{1}\right) P_{1}\left(s_{2}\right)$, and Eq. (18) reduces to a product of two terms, each being of the form (19). This transparent physical picture underlying the multiplication rule is particularly helpful if one seeks to understand the role of losses (absorption and/or leakage of light) at the inter-layer interface. Increasing the losses makes the partial lengths of light paths $s_{1}$ and $s_{2}$ in the layers more and more independent, since the losses reduce the probability for a typical path to cross the interface more than once. Consequently, most of the paths will consist of two independent segments, one inside the first layer (length $s_{1}$ ), and the other one - inside the second layer $\left(s_{2}\right)$. $P_{2}\left(s_{1}, s_{2}\right)$ will then reduce to a product 
$P_{1}\left(s_{1}\right) P_{1}\left(s_{2}\right)$, exactly as required for the multiplication rule to hold. Note that the Siegert relation (12) implies that the multiplication rule applies to $g_{2}(\tau)-1$ as well.

\section{EXPERIMENTAL SETUP}

Our experimental setup consists of a frequencydoubled NdYAG laser ("Verdi" from Coherent, wavelength $\lambda_{0}=532 \mathrm{~nm}$ ) with a beam width of roughly 1 $\mathrm{mm}$. Only minor differences are found upon expanding the beam to $7 \mathrm{~mm}$ in width (see also Ref. [7] ). The laser radiation is directed at the surface of the sample consisting of two cells, and the multiple-scattered, transmitted light is collected using a single mode optical fiber. The intensity of collected light is analyzed by a digital correlator (see Fig. 31). Apart from the complex structure of the sample, the described experimental setup represents a classical one for diffusing-wave spectroscopy experiments in transmission geometry [2] 5]. We pay special attention to the preparation of the sample, which is a key feature of our experiments. The first cell (thickness $L_{1}$ ) is filled by a colloidal gel, prepared from a destabilized solution of polystyrene spheres (diameter $170 \mathrm{~nm}$ ) at a volume fraction up to $20 \%$ in a buoyancy-matching mixture of water and heavy water [11,67]. In our experiments, the gel serves as a model nonergodic medium where the average mean square displacement of the constituent particles is reasonably well described by the model of arrested subdiffusive motion [see Eq. (3)]. In general, gel systems undergo a complicated temporal evolution (see, e.g., Refs. [11, 13 15, 50,54,66]), which is not a subject of the present paper. For our measurements, we wait after the sol-gel transition till the height of the plateau of $g_{2}(\tau)-1$ is of the order 0.5 , and the gel properties remain constant over the measurement time of about $10-30 \mathrm{~min}$. We note that experimentally $g_{2}(\tau)-1$ does not exhibit a perfect plateau but rather a long stretching. This indicates a second, long-time decay of the autocorrelation function, not included in our simple theoretical model [Eq. (3)]. The characteristic time scale of this decay extends to minutes or hours 11,54, and hence is beyond the time window of our experiments.

Two different realizations of the two-cell sample were used in the experiments reported below (samples A and B for brevity). While sample A is a model sample constructed to test the validity of our approach, sample B is optimized to facilitate its experimental application (the multiplication rule holds for this sample).

Sample A. The sample consists of three disc-shaped microscope cover plates (radius $R=7 \mathrm{~mm}$, thickness $\Delta=0.15 \mathrm{~mm})$ and two ring-shaped teflon spacers. The first cell contains a colloidal gel (volume fraction $\Phi=$ $7 \%$, photon transport mean free path $\left.l_{1}^{*}=53 \mu \mathrm{m}\right)$. The second cell is filled with a suspension of $\mathrm{TiO}_{2}$ powder (Aldrich, particle diameter $<5 \mu \mathrm{m}$ ) dispersed in pure glycerol (refractive index $n=1.47$ ).

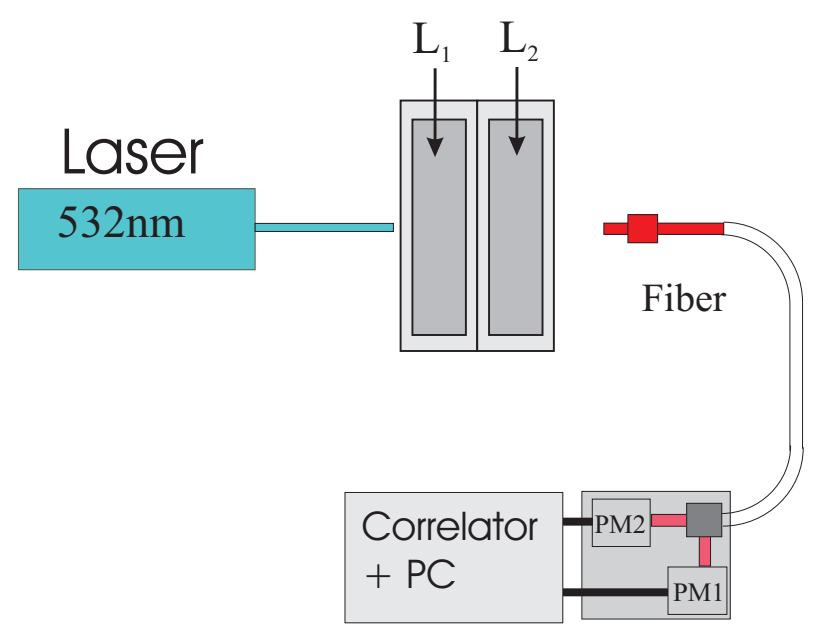

FIG. 3. Experimental setup. A laser beam $\left(\lambda_{0}=532 \mathrm{~nm}\right)$ is incident on a sandwich of two turbid layers. The light transmitted through the sandwich is detected with a mono-mode fiber, and is subsequently analyzed with a photomultiplier $(\mathrm{PM}) /$ digital correlator unit.

The transport mean free path for this suspension is $l_{2}^{*}=41 \mu \mathrm{m}$. The single scattering correlation time corresponding to the suspension in the second cell is $\tau_{0}=23 \pm 1 \mathrm{~s}$, as determined by standard DWS measurements in backscattering geometry [3]. The thicknesses $L_{1}$ and $L_{2}$ of the layers were determined from DWS measurements in transmission geometry by filling them with a suspension of known $l^{*}$ and fitting the resulting intensity autocorrelation functions with Eq. (13). We find $L_{1}=1.75 \mathrm{~mm}$ and $L_{2}=1.9 \mathrm{~mm}$. The optical thicknesses of both layers are comparable and high enough $\left(L_{1} / l_{1}^{*} \simeq 33 \sim L_{2} / l_{2}^{*} \simeq 46 \gg 1\right)$, and therefore we call this sample "symmetric". A typical photon path length $s$ in the sample is $s \sim L^{2} / l^{*} \approx 4 \times 10^{2} \mathrm{~mm}$ (with $L \simeq L_{1}+L_{2} \approx 4 \mathrm{~mm}, l^{*}=l_{1}^{*} \approx l_{2}^{*} \approx 40 \mu \mathrm{m}$ ), which is two orders of magnitude larger than the sample thickness. Hence, the scattering of light in our sample is essentially multiple, and a typical photon path is expected to cross the buried inter-layer interface many times. Estimation [72] of the attenuation factor $f$ gives $f \simeq 0.98 \simeq 1$, and hence the multiplication rule will only hold for the sample A in a trivial situation of $\alpha_{1} L_{1} \ll 1$ and $\alpha_{2} L_{2} \ll 1$, as $L_{1} / l_{1}^{*} \sim L_{2} / l_{2}^{*}$.

Sample B. The sample consists of two equally thick light-scattering cells (Hellma, $L_{1}=L_{2}=1 \mathrm{~mm}$ ). The thickness of the glass wall between the turbid media inside the cells is $\Delta \simeq 2 \mathrm{~mm}$. Such a thick wall leads to a significant photon leakage in transverse directions, and we put an aperture of radius $R=2.5 \mathrm{~mm}$ in between the two cells to prevent the photons leaked out of the cell from reaching the detector. This leads to some additional decrease of the total transmitted intensity, while ensures the multiplication rule, as discussed in Sec. IV. Indeed, for $f \simeq 0.46$ estimated theoretically [72] the multiplication rule should hold whenever $L_{n} / l_{n}^{*} \gg 1 /\left(1-f^{2}\right) \sim 1$ 
and $\alpha_{n} \ell_{n}^{*} \ll 1-f^{2} \sim 1$, which coincides with the conditions of validity of Eq. (11). We expect the actual value of $f$ to be even smaller due to (total) reflections of light at the interface which are not included in our present theoretical model. Even though the conditions of validity of the multiplication rule are satisfied, to apply it in a real experiment [i.e. to find $g_{2}^{(1)}\left(L_{1}, \tau\right)-1$ as a ratio of $g_{2}^{(2)}\left(L_{1}, L_{2}, \tau\right)-1$ and $\left.g_{2}^{(1)}\left(L_{2}, \tau\right)-1\right]$ we need both $g_{2}^{(2)}\left(L_{1}, L_{2}, \tau\right)-1$ and $g_{2}^{(1)}\left(L_{2}, \tau\right)-1$ to be essentially different from 0 , as otherwise experimental errors in determination of the above autocorrelation functions can be significant, making the application of the multiplication rule impractical. To overcome this problem, we choose the second light-scattering cell to be of much smaller optical thickness than the first one, while the motion of scatterers is made slow inside it. Namely, the first cell is filled with a very turbid gel (volume fraction $\Phi=20 \%$, $l_{1}^{*}=19 \mu \mathrm{m}$ ), while the second one contains a moderately turbid suspension of colloidal polystyrene dispersed in a water-glycerol mixture $\left(l_{2}^{*}=300-500 \mu \mathrm{m}\right)$. The decrease of the measured autocorrelation function due to the motion of scatterers in the second, ergodic cell is thereby shifted to long times $\tau \sim \tau_{2}=\tau_{0}\left(l_{2}^{*} / L_{2}\right)^{2}$ and does not obscure the light-scattering signal of the first, nonergodic cell. Hence, the multiplication rule can be efficiently applied for $\tau \lesssim \tau_{2}$, while for $\tau \gg \tau_{2}$ the measured autocorrelation function will decrease to 0 due to the motion of scatterers in the second cell. For the sample $\mathrm{B}, L_{1} / l_{1}^{*} \approx 50 \gg L_{2} / l_{2}^{*} \approx 2-3$, and therefore we call this sample "asymmetric".

\section{RESULTS AND DISCUSSION}

In order to demonstrate the efficiency of the method proposed and theoretically justified in Secs. II III, and to show the feasibility of diffusing-wave spectroscopy in nonergodic media, we have carried out several model experiments using samples A (symmetric) and B (asymmetric).

\section{A. Symmetric sample}

Open circles in Fig. 1(a) show the intensity autocorrelation function $g_{2}^{(2)}\left(L_{1}, L_{2}, \tau\right)-1$ of light transmitted through the symmetric sample A. The solid line going through the open circles of Fig. 1 (a) is obtained by fitting the experimental data with Eq. (11) assuming $f=1$ (no leakage of light at the wall separating the cells). The fitting parameters are the gel parameters $\delta=2.24 \mathrm{~nm}$, $\tau_{c}=25 \mu \mathrm{s}, p=0.6$ [see Eq. (3)], while the parameters of the medium in the second cell, as well as the cell thicknesses $L_{1}$ and $L_{2}$ were determined independently. We note that the two sets of parameters are only weakly correlated in the fit. Theory and experimental data are found in excellent agreement.
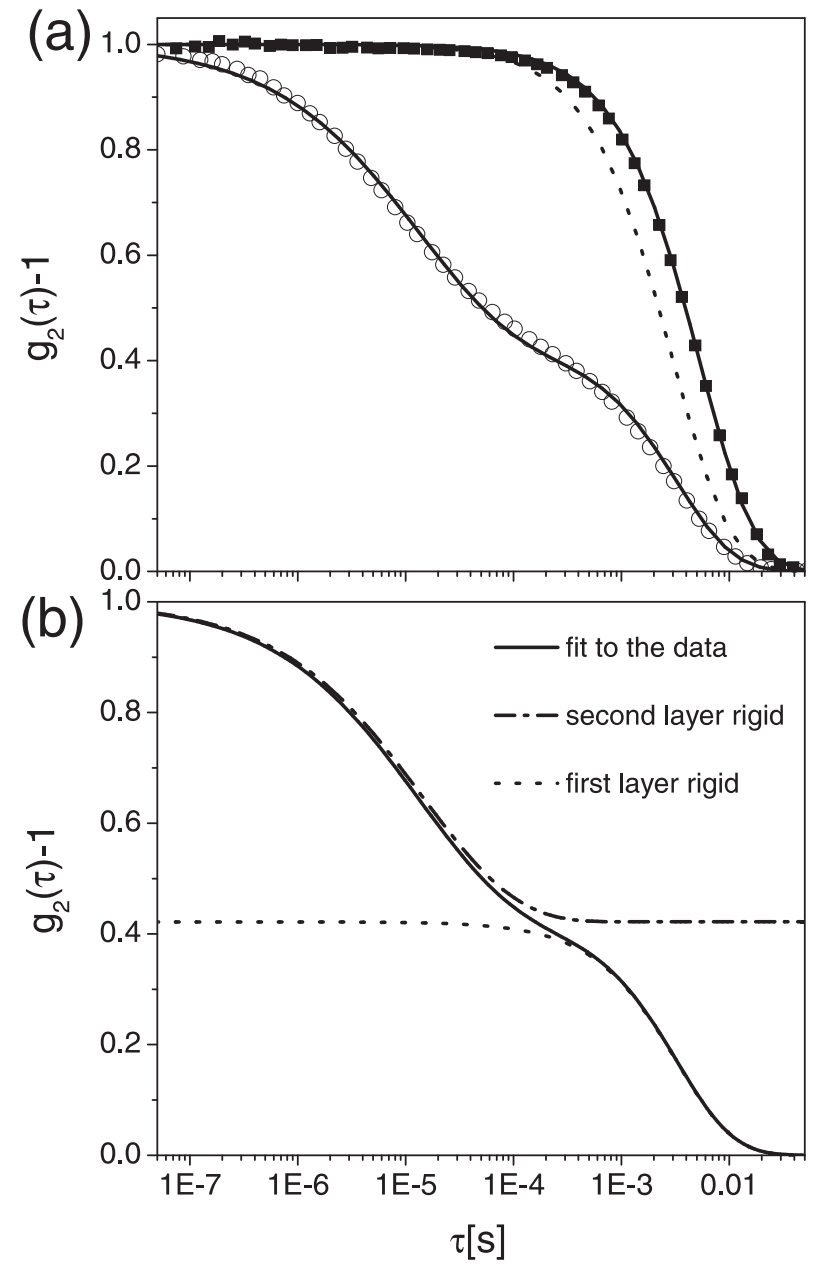

FIG. 4. (a) Intensity autocorrelation function for transmission through the symmetric two-cell sample A (open circles), and through the isolated second cell (full squares). Theoretical fits [Eq. (11) with $f=1$ and Eq. (13)] are shown by solid lines. The dotted line shows the theoretical correlation function of the two-cell sample with the first layer assumed to be rigid $\left(\tau_{c} \rightarrow \infty\right)$. (b) Different contributions to the intensity autocorrelation function are shown. The solid line is the fit to the two-cell data of the panel (a). To obtain the dashed-dotted line, we keep the second layer rigid $\left(\tau_{0} \rightarrow \infty\right)$, while all other parameters are unchanged. The dotted line is that of the panel (a) multiplied by the plateau value 0.422 .

As shown in Fig. $1(\mathrm{a})$, the autocorrelation function of the two-cell setup $g_{2}^{(2)}\left(L_{1}, L_{2}, \tau\right)-1$ exhibits two characteristic decay times. The fast decay is due to the gel in the first layer, while the slow decay corresponds to the dynamics in the second layer. This is illustrated in Fig. 四(b), where we decompose $g_{2}^{(2)}\left(L_{1}, L_{2}, \tau\right)-1$ in two contributions due to the dynamics in the first and second layers, respectively. The contributions of the first (dashdotted line) and second (dotted line) layers are obtained by assuming the second or the first layer to be rigid [Eq. (11) with $f=1$ and $\alpha_{2} \equiv 0$ or $\alpha_{1} \equiv 0$, respectively]. The contribution of the second layer is renormalized so 
that its value at $\tau=0$ is equal to the contribution of the first layer at $\tau \rightarrow \infty$. The figure suggests that a simple interpretation of the correlation function resulting from the two-cell setup in terms of contributions of individual layers can be given if the decay times due to different layers are well separated.

The dotted line in Fig. 目(a) shows the contribution of the second layer to $g_{2}^{(2)}\left(L_{1}, L_{2}, \tau\right)-1$ [the same as in Fig. [(b) but renormalized to 1]. Due to the presence of the first layer, although assumed to be rigid, the decay of the autocorrelation function becomes significantly faster as compared to the second layer taken alone [full squares in Fig. [1(a)]. This stems from the fact that the typical lengths of photon paths in the second layer are increased due to the presence of the first layer, which acts as an effective "diffuse mirror", increasing the probability for a light path to get back to the second layer instead of being diffusely reflected from the sample.

The measurements performed on the symmetric sample A allows us to conclude that the multiple scattering of light in a two-cell sample is correctly described by the theory developed in Sec. III. At the same time, the results of this subsection serve as a test of our method, allowing diffusing-wave spectroscopy to be applied to nonergodic random media. Indeed, the parameters of the (nonergodic) gel $\left(\delta, \tau_{c}\right.$ and $\left.p\right)$ can be obtained from the fit to the experimental data [see Fig. 团, provided that the parameters of the medium in the second cell as well as the thicknesses of both cells are measured independently.

\section{B. Asymmetric sample}

Although the results of the previous subsection seem to be sufficiently convincing to justify our method of performing DWS in nonergodic media, we will now show that the interpretation of experimental data can be further simplified by introducing leakage (or absorption) of light at the interface between the layers in combination with a proper choice of parameters of the second layer. An optimal choice of the parameters corresponds to our asymmetric sample B (see Sec. V). For this sample, the optical thickness of the second layer is much smaller than that of the first one, and the leakage of light at the interlayer wall is considerable. This ensures the validity of the multiplication rule formulated in Sec. IV], as confirmed by the experimental results presented below. Figures 5(a) and (b) show the intensity autocorrelation functions $g_{2}^{(2)}\left(L_{1}, L_{2}, \tau\right)-1$ obtained for the two-cell setup (open circles). The results for two different second cells are displayed. Full squares show $g_{2}^{(1)}\left(L_{2}, \tau\right)-1$ obtained for the isolated second cells, just as in Fig. A. The corresponding single scattering correlation time $\tau_{0}=\left(k^{2} D_{B}\right)^{-1}$ of the particles in the second cell has been varied by changing the glycerol content in the suspending liquid, which affects the viscosity of the liquid and, consequently, the particle diffusion coefficient $D_{B}$.

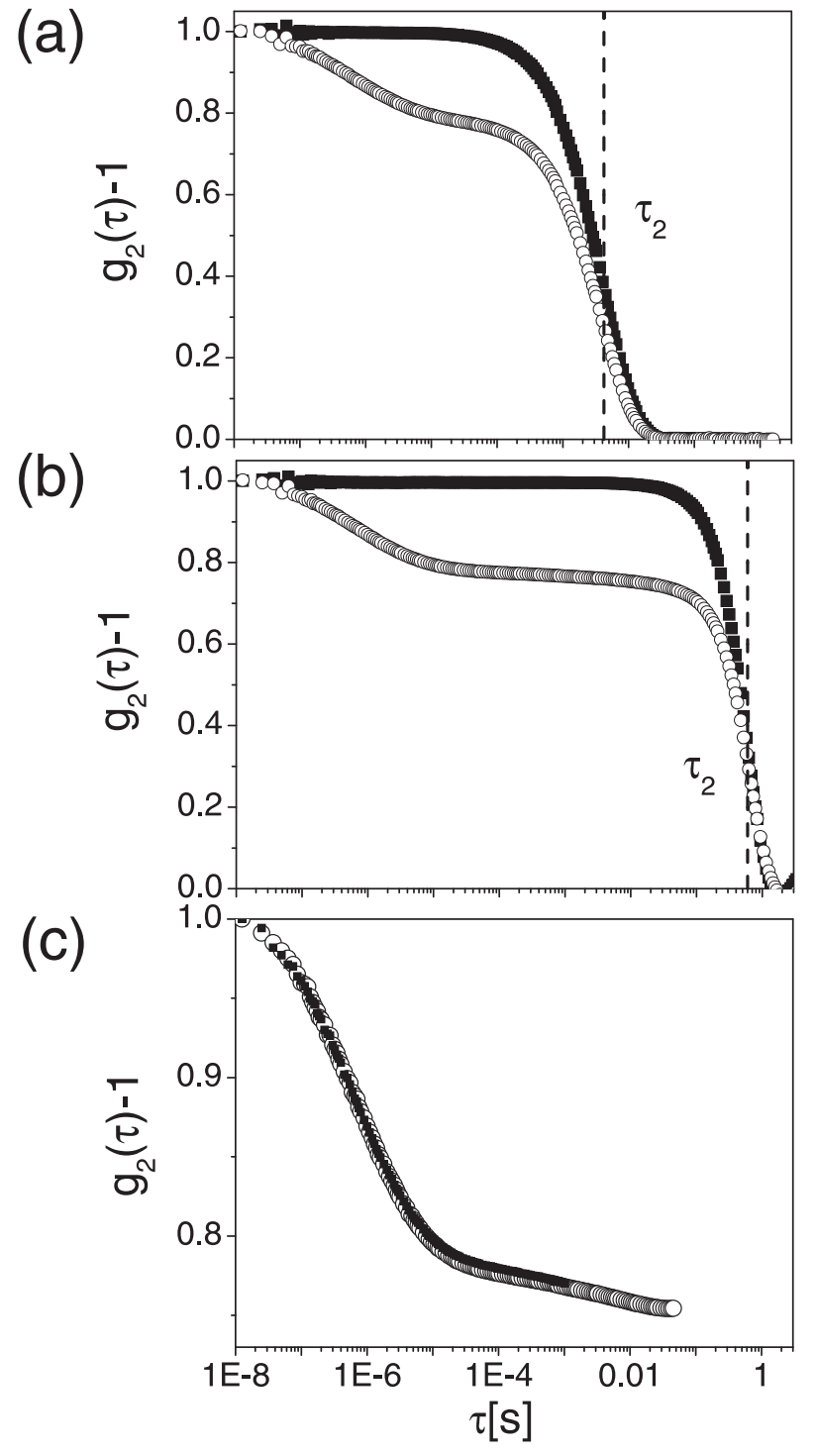

FIG. 5. Multiplication rule: (a) Intensity autocorrelation function measured in transmission through the asymmetric two-cell sample B (open circles). Full squares show the autocorrelation function measured for the isolated second cell [decay time $\tau_{2}=\tau_{0}\left(l_{2}^{*} / L_{2}\right)^{2}$ ]. (b) Same as (a), but for a different medium inside the second cell (higher glycerol content of the suspending liquid leading to a reduced particle diffusion coefficient and a larger value of $\tau_{2}$ ). (c) The ratios of the autocorrelation functions are shown for the data of panels (a) (open circles) and (b) (full squares). The results are identical for $\tau<5 \tau_{2}$ [with $\tau_{2}$ of panel (a)], and yield the ensemble-averaged intensity autocorrelation function $g_{2}^{(1)}\left(L_{1}, \tau\right)-1$ corresponding to the nonergodic first cell taken alone. Inset: Relative difference $\Delta(\tau)=\left\{\left[g_{2}^{(a)}(\tau)-1\right]-\left[g_{2}^{(b)}(\tau)-1\right]\right\} /\left[g_{2}^{(a)}(\tau)-1\right]$ between the two correlation functions of the main plot.

According to the multiplication rule [Eq.(16)], the ratio $\left[g_{2}^{(2)}\left(L_{1}, L_{2}, \tau\right)-1\right] /\left[g_{2}^{(1)}\left(L_{2}, \tau\right)-1\right]$ is expected to be equal to $g_{2}^{(1)}\left(L_{1}, \tau\right)-1$, i.e. the autocorrelation function corresponding to the (first) nonergodic light-scattering cell. 
To check this prediction, we have calculated corresponding ratios for the curves of Figs. $5(a)$ and (b). The results are presented in Fig. 5(c) [full squares correspond to the data of Fig. 5(a), while open circles - to the data of Fig. 5(b)]. The two curves are indistinguishable, supporting the validity of the multiplication rule. Experimentally, we find that for $\tau<5 \tau_{2}$ [with $\left.\tau_{2}=\tau_{0}\left(l_{2}^{*} / L_{2}\right)^{2}\right]$ the deviations from the multiplication rule are negligible. The perfect agreement of the results obtained for two different media in the second cell confirms the validity of our method.

As an additional check of the multiplication rule, we have performed measurements of the intensity autocorrelation function $g_{2}^{(2)}\left(L_{1}, L_{2}, \tau\right)-1$ with the first layer of the double-layer sample being totally rigid (white paper was used to model rigid but turbid random medium). The results are presented in Fig. 6 by open circles for $g_{2}^{(2)}\left(L_{1}, L_{2}, \tau\right)-1$ and full squares for $g_{2}^{(1)}\left(L_{2}, \tau\right)-1$ (the latter is measured for the isolated second layer). Full circles are obtained by calculating the ratio $\left[g_{2}^{(2)}\left(L_{1}, L_{2}, \tau\right)-\right.$ $1] /\left[g_{2}^{(1)}\left(L_{2}, \tau\right)-1\right]$. As expected, the ratio is one over a sufficiently extended time range (the ratio one corresponds to no scatterer motion in the paper).

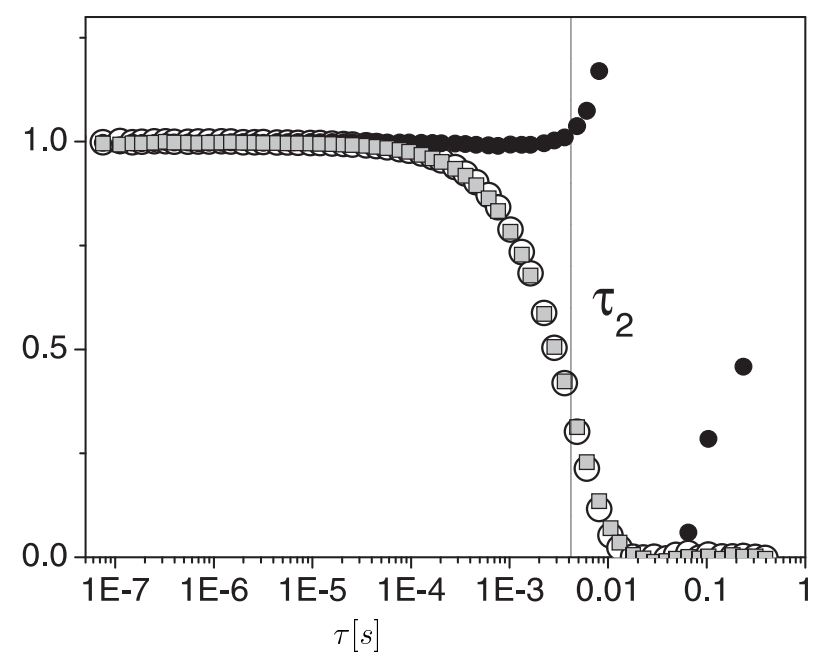

FIG. 6. Intensity autocorrelation function for a two-cell sample with a rigid first layer (the colloidal gel in the first layer was replaced by a white paper which mimics a rigid turbid medium) (open circles). Full squares show the autocorrelation function corresponding to the isolated second layer. The ratio of the two autocorrelation functions (full circles) is one to a good accuracy for $\tau<\tau_{2}=\tau_{0}\left(l_{2}^{*} / L_{2}\right)^{2}$, corresponding to the absence of scatterer motion in the first layer.

\section{HOW TO APPLY THE TWO-CELL TECHNIQUE ?}

One of the major advantages of the two-cell technique is its simplicity. Nevertheless, we would like to point out some pitfalls when designing a two-cell experiment. Firstly, it is worthwhile to mention the problem of low transmitted light intensity. Building a sandwich with regular glass cuvettes can result in a sample of significant overall thickness. Light incident on the setup spreads out diffusely which leads to relatively low intensity of light reaching the transmission side. The problem becomes even more severe if absorption and/or leakage of light are introduced at the inter-cell wall to simplify the interpretation of experimental data by using the multiplication rule. Secondly, due to reflections and additional leakage of diffuse light at the interface glass walls, some of the light scattered out of the cell can reach the detector without passing through the whole sample. To avoid this problem, we recommend to put an aperture (e.g. a black plastic foil with a centered hole of typically $5 \mathrm{~mm}$ in diameter) between the cells to suppress photons scattered snake-like along the cell walls. Ideally, the double-cell sandwich would have only one moderately absorbing thin interface wall with a built-in circular aperture forcing all transmitted light to pass through the whole sample.

\section{SUMMARY AND OUTLOOK}

In the present paper we propose and test experimentally a new method for the application of diffusing-wave spectroscopy to nonergodic turbid media. We show that light transmitted trough a sandwich of two turbid samples can be considered ergodic even if only the second sample is ergodic. The autocorrelation function of the transmitted intensity can be quantitatively described by the diffusion theory. This allows direct application of diffusing-wave spectroscopy for the characterization of nonergodic media without any additional efforts usually required to achieve ensemble averaging (i.e. without translation or rotation of the sample in course of the correlation function measurement [29, 34, 35, 50]). Proper averaging of the light-scattering signal is ensured by adding a second, ergodic light-scattering cell with unprecedented accuracy. In order to simplify the analysis of the experimental data, the parameters of the doublecell sample can be optimized. Namely, moderate absorption and/or leakage of light should be introduced at the interface between the light-scattering cells, and the optical thickness of the second, ergodic cell should be reduced well below the optical thickness of the cell containing the nonergodic medium, while the dynamics of scatterers in the second cell should be chosen slow. Under these conditions we have shown that the field autocorrelation function of light transmitted through the double-layer sample can be written as a product of autocorrelation functions corresponding to the individual layers: $g_{1}^{(2)}\left(L_{1}, L_{2}, \tau\right)=g_{1}^{(1)}\left(L_{1}, \tau\right) \times g_{1}^{(1)}\left(L_{2}, \tau\right)$. Consequently, it is sufficient to measure the intensity autocorrelation functions [56] of the two cell setup $g_{2}^{(2)}\left(L_{1}, L_{2}, \tau\right)$ and of the second, ergodic layer $g_{2}^{(1)}\left(L_{2}, \tau\right)$, in order 
to obtain the properly averaged intensity autocorrelation function of the nonergodic layer $g_{2}^{(1)}\left(L_{1}, \tau\right)-1=$ $\left[g_{2}^{(2)}\left(L_{1}, L_{2}, \tau\right)-1\right] /\left[g_{2}^{(1)}\left(L_{2}, \tau\right)-1\right]$. Henceforth application of diffusing-wave spectroscopy for the characterization of nonergodic media becomes straightforward. Our method is particularly suited for the study of time evolving systems, e.g. aggregating and gelling particle suspensions (see also Refs. [11] and [15]), since the data acquisition time can easily be adjusted from a few seconds to several hours. Other promising applications include DWS-microrheology which has previously been limited to time and length scales where the motion of tracer particles is not significantly constrained [36, 37]. We expect the two-cell technique to extend the measurement range of this method and to provide access to a novel class of (solid-like) materials.

\section{ACKNOWLEDGMENTS}

We thank Veronique Trappe for useful comments and discussions.

[1] G. Maret and P. E. Wolf, Z. Phys. B 65, 409 (1987).

[2] D. J. Pine, D. A. Weitz, P. M. Chaikin, and E. Herbolzheimer, Phys. Rev. Lett. 60, 1134 (1988).

[3] D. J. Pine, D. A. Weitz, G. Maret, P. E. Wolf, E Herbolzheimer, and P. M. Chaikin, in: Scattering and Localization of Classical Waves in Random Media, ed. P. Sheng (World Scientific, Singapore, 1989), p. 312.

[4] D. J. Pine, D. A. Weitz, J. X. Zhu, and E. Herbolzheimer, J. Phys. France 51, 2101 (1990).

[5] D. A. Weitz and D. J. Pine, in Ref. [9], p. 652.

[6] D. A. Weitz, J. X. Zhu, D. J. Durian, H. Gang, and D. J. Pine, Phys. Scr. T49B, 610 (1993).

[7] P. D. Kaplan, A. D. Dinsmore, A. G. Yodh, and D. J. Pine, Phys. Rev. E. 50, 4827 (1994).

[8] B. J. Berne and R. Pecora, Dynamic Light Scattering (Wiley, New York, 1976).

[9] Dynamic Light Scattering, ed. W. Brown (Oxford University Press, New York, 1993).

[10] G. Maret, Curr. Opin. Coll. Int. Sci. 2, 251 (1997).

[11] S. Romer, F. Scheffold, and P. Schurtenberger, Phys. Rev. Lett. 85, 4980 (2000).

[12] P. D. Kaplan, A. G. Yodh and D. F. Townsend, J. Coll. Int. Sci 155, 319 (1993).

[13] C. Urban, S. Romer, F. Scheffold, and P. Schurtenberger, Progr. Colloid Polym. Sci. 115, 270 (2000).

[14] C. Urban, S. Romer, and P. Schurtenberger, in Proceedings of the 2nd International Symposium on Food Rheology and Structure, P. Fischer, I. Marti, and E. J. Windhab, Eds., (Zurich, 2000), p. 41.

[15] H. Wyss, S.Romer, F. Scheffold, P. Schurtenberger, and L. J. Gauckler, submitted.
[16] H. Gang, A. H. Krall, and D. A. Weitz, Phys. Rev. E 52, 6289 (1995).

[17] P. Hebraud, F. Lequeux, J. P. Munch, and D. J. Pine, Phys. Rev. Lett. 78, 4657 (1997).

[18] V. Lisy and B. Brutovsky, Phys. Rev. E 59, 3765 (1999) (comment on Ref. 16]).

[19] D. J. Durian and D. A. Weitz, Science 252, 686 (1991); D. J. Durian, D. A. Weitz, and D. J. Pine, Phys. Rev. A 44, R7902 (1991).

[20] J. C. Earnshaw and A. H. Jaafar, Phys. Rev. E 49, 5408 (1994).

[21] A. D. Gopal and D. J. Durian, Phys. Rev. Lett. 75, 2610 (1995).

[22] R. Höhler, S. Cohen-Addad, and H. Hoballah, Phys. Rev. Lett. 79, 1154 (1997).

[23] A. D. Gopal and D. J. Durian, J. Opt. Soc. Am. A 14, 150 (1997).

[24] N. Menon and D. J. Durian, Phys. Rev. Lett. 79, 3407 (1997); Science 275, 1920 (1997).

[25] D. J. Durian, J. Phys. C: Cond. Matt. 12, A507 (2000).

[26] D. A. Boas, G. Nishimura, and A. G. Yodh, Proc. SPIE 2979, 468 (1997).

[27] A. Palmer, T. G. Mason, X. Jingyuan, S. C. Kuo, and D. Wirtz, Biophys. J. 76, 1063 (1999).

[28] R. Lohwasser and G. Soelkner, Appl. Opt. 38, 2128 (1999).

[29] D. A. Boas, L. E. Campbell, and A. G. Yodh, Phys. Rev. Lett. 75, 1855 (1995).

[30] M. Heckmeier and G. Maret, Europhys. Lett. 34, 257 (1996).

[31] S. E. Skipetrov and R. Maynard, Phys. Lett. A 217, 181 (1996).

[32] M. Heckmeier, S. E. Skipetrov, G. Maret, and R. Maynard, J. Opt. Soc. Am. A 14, 185 (1997).

[33] D. A. Boas and A. G. Yodh, J. Opt. Soc. Am. A 14, 192 (1997).

[34] S. E. Skipetrov and I. V. Meglinskii, Zh. Éksp. Teor. Fiz. 113, 1213 (1998) [JETP 86, 661 (1998)].

[35] M. Heckmeier and G. Maret, Progr. Colloid Polym. Sci. 104, 12 (1997); Opt. Commun. 148, 1 (1998).

[36] T. G. Mason and D. A. Weitz, Phys. Rev. Lett. 74, 1250 (1995).

[37] T. Gisler and D. Weitz, Curr. Opin. Coll. Int. Sci. 3, 586 (1998).

[38] J. C. Crocker, M. T. Valentine, E. R. Weeks, T. Gisler, P. D. Kaplan, A. G. Yodh, and D. A. Weitz, Phys. Rev. Lett. 85, 888 (2000).

[39] T. G. Mason, T. Gisler, K. Kroy, E. Frey, and D. A. Weitz, J. Rheol., to appear (2000).

[40] D. A. Weitz, D. J. Pine, P. N. Pusey, and R. J. A. Tough, Phys. Rev. Lett. 63, 1747 (1989).

[41] D. J. Pine, X. L. Wu, P. M. Chaikin, and D. A. Weitz, J. Opt. Soc. Am. B 7, 15 (1990).

[42] D. Bicout, E. Akkermans, and R. Maynard, J. Phys. I (Paris) 1, 471 (1991).

[43] D. Bicout and R. Maynard, Physica A 199, 387 (1993).

[44] D. J. Bicout and R. Maynard, Physica B 204, 20 (1995).

[45] S. E. Skipetrov and R. Maynard, Phys. Rev. Lett. 85, 736 (2000).

[46] S. E. Skipetrov, Phys. Rev. E., to appear (2001); see also 
cond-mat/0101059.

[47] P. N. Pusey and W. van Megen, Physica A 157, 705 (1989).

[48] J. G. H. Joosten, E. T. F. Geladé, and P. N. Pusey, Phys. Rev. A 42, 2161 (1990).

[49] W. van Megen, S. M. Underwood, and P. N. Pusey, Phys. Rev. Lett. 67, 1586 (1991).

[50] J. Z. Xue, D. J. Pine, S. T. Milner, X.-l. Wu, and P. M. Chaikin, Phys. Rev. A 46, 6550 (1992).

[51] K. Schatzel, Appl. Opt. 32, 3880 (1993).

[52] S. Kirsch, V. Frenz, W. Schartl, E. Bartsch, and H. Sillescu, J. Chem. Phys. 104, 1758 (1996).

[53] E. Bartsch, V. Frenz, J. Baschnagel, W. Schartl, and H. Sillescu, J. Chem. Phys. 106, 3743 (1997).

[54] L. Cipelletti, S. Manley, R.C. Ball, and D. A. Weitz, Phys. Rev. Lett. 84, 2275 (2000).

[55] A patent application for this method was filed with the Suisse Institute of Intellectual Property on February 27, 2000 under number 200 0335/00.

[56] The field and intensity autocorrelation functions of multiple-scattered waves are directly related through the Siegert relation, see Ref. 8] and Eq. (12).

[57] H. Gang, A. H. Krall, H. Z. Cummins and D. A. Weitz, Phys. Rev. E. 59, 715 (1999).

[58] G. Nisato, P. Hebraud, J.-P. Munch, and S. J. Candau, Phys. Rev. E 61, 2879 (2000).

[59] A. Knaebel, M. Bellour, J.-P. Munch, V. Viasnoff, F. Lequeux, and J. L. Harden, Europhys. Lett. 52, pp. 73$79(2000)$

[60] J. X. Zhu, D. J. Pine, and D. A. Weitz, Phys. Rev. A 44, 3948 (1991).

[61] R. C. Haskell, L. V. Swaasand, T. Tsay, T. Feng, M. S. McAdams, and B. J. Tromberg, J. Opt. Soc. Am. A 11, 2727 (1994).

[62] R. Aronson, J. Opt. Soc. Am. A 12, 2532 (1995).

[63] N. G. Chen and J. Bai, Phys. Rev. Lett. 80, 5321 (1998).

[64] M. U. Vera and D. J. Durian, Phys. Rev. E 53, 3215 (1996).

[65] We note that in the case of relatively small particles $\left(k_{0} d \approx 1\right)$ structural correlations $S(q)$ and hydrodynamic interactions $h(q)$ have to be taken into account if present. This complicates a quantitative determination of the particle mean square displacement significantly. For details see Ref. [5].

[66] A. H. Krall and D. A. Weitz, Phys. Rev. Lett. 80, 778 (1998).

[67] T. J. Graule, F. H. Baader, and L. J. Gauckler, J. Mater. Educ. 16, 243 (1994)

[68] S. Fraden and G. Maret, Phys. Rev. Lett. 65, 512 (1990); X. Qiu, X. L. Wu, J. Z. Xue, D. J. Pine, D. A. Weitz and P. M. Chaikin, Phys. Rev. Lett. 65, 516 (1990).

[69] B. Shapiro, Phys. Rev. Lett. 57, 2168 (1986).

[70] J. Ripoll, M. Nieto-Vesperinas, S. R. Arridge, and H. Dehghani, J. Opt. Soc. Am. A 17, 1671 (2000).

[71] See Eq. (36) of Ref. [70, where we replace $U_{1}(\mathbf{r})$ by $G_{1}(\mathbf{r}, \tau) \equiv G_{1}(z, \tau)$ and introduce $f(\mathbf{r})=(1 / \pi) \int_{S} \mathcal{G}(\mathbf{r}-$ $\left.\mathbf{r}^{\prime}\right) \mathrm{d} S^{\prime}$, where the integration is over one of the surfaces $\left(z=L_{1}\right.$ or $\left.z=L_{1}+\Delta\right)$ of the nonscattering wall separating the turbid layers, and $\mathcal{G}\left(\mathbf{r}-\mathbf{r}^{\prime}\right)$ is defined by Eq. (18) of Ref. 70].
[72] We neglect the absorption of light in the glass wall, and assume that $f$ is mainly determined by the leakage of light in transverse directions [see Eq. (10)]. 